\title{
Eosinophilic Granuloma Associated with Intratumoral Hemorrhage
}

\author{
-Case Report-
Ryunosuke URANISHI, Yuji NIKAIDO, Takahiko EGUCHI, Hironobu BESSHO, Takatoshi FuJIMOTO, and Takuo INUI \\ Department of Neurosurgery, Osaka-Minami National Hospital, \\ Kawachinagano, Osaka

\begin{abstract}
A 2-year-old boy presented with a rapidly growing soft scalp mass in the left parietal region. Surgical exploration and histological examination demonstrated an eosinophilic granuloma associated with intratumoral hemorrhage. Eosinophilic granuloma should also be considered when a scalp mass lesion exhibits rapid growth.
\end{abstract}

Key words: eosinophilic granuloma, hemorrhage, skull tumor

\section{Introduction}

Histiocytosis $\mathrm{X}$ is a clinical entity characterized by proliferation of Langhans' giant cells, and includes three distinct diseases: eosinophilic granuloma, Hand-Schüller-Christian disease, and Letterer-Siwe disease. ${ }^{5)}$ Letterer-Siwe disease is presently considered to be a different clinical entity because of its aggressive and systemic behavior. ${ }^{4)}$ Eosinophilic granuloma primarily occurs in children and young adults, and is predominantly located in the skull. ${ }^{6,8,9)}$ Eosinophilic granuloma usually develops as a solitary lesion, but may occur at multiple sites. ${ }^{97}$ This lesion is benign and usually enlarges gradually. The occurrence of intratumoral hemorrhage in eosinophilic granulomas has never been reported.

We present a case of an eosinophilic granuloma in the parietal region associated with intratumoral hemorrhage.

\section{Case Report}

A 2-year-old boy was admitted to our hospital with a mass in the left parietal region. His mother had first noticed the $1 \times 1-\mathrm{cm}$ mass 1 month before admission, and the mass had since enlarged rapidly. Physical examination on admission was unremarkable except for the non-tender soft mass with some fluctuation in the left parietal region. The size of the lesion was $3 \times 3 \mathrm{~cm}$. There was no change in size while sitting or standing.

Skull radiography showed a radiolucent lytic lesion in the left parietal bone without a sclerotic margin (Fig. 1). Computed tomography (CT) revealed an isodense mass and destruction of both inner and outer tables (Fig. 2 left). The mass was homogeneously enhanced by administration of contrast material (Fig. 2 right). Magnetic resonance (MR) imaging disclosed a slightly hyperintense lesion on the $T_{1}$ weighted image in the left parietal bone, and small low intensity core in a high intensity area on the $\mathrm{T}_{2-}$ weighted image (Fig. 3 left, center). $\mathrm{T}_{1}$-weighted $\mathrm{MR}$ imaging after administration of gadoliniumdiethylenetriaminepenta-acetic acid (Gd-DTPA) revealed ring-like enhancement of the lesion which compressed the underlying dura slightly (Fig. 3

Received September 11, 1995; Accepted February 22, 1996

Author's present address: R. Uranishi, M.D., Department of Neurosurgery, Nara Medical University, Kashihara, Nara, Japan. 


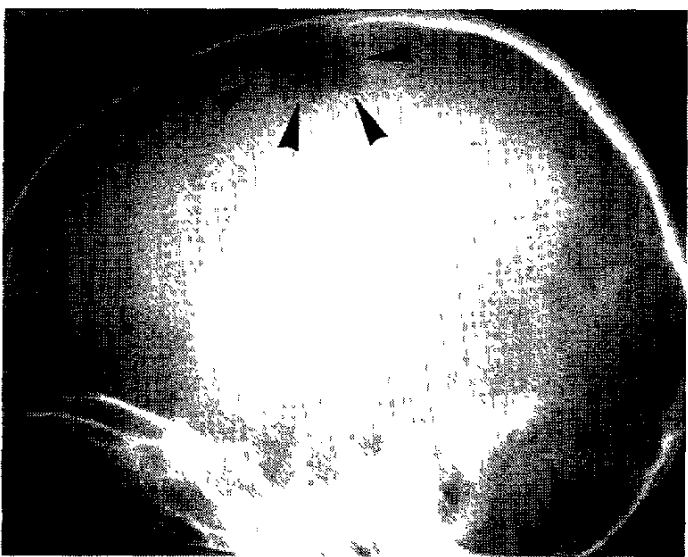

Fig. 1 Skull radiograph showing an osteolytic radiolucent area (arrowheads) with a slightly irregular margin in the left parietal bone.

right). Cerebral angiography did not demonstrate a tumor stain or abnormal vessels.

Radical surgery was performed under general anesthesia. A half-circle shaped skin incision surrounding the tumor was made, then the skin flap was reflected subgaleally. A reddish-gray soft tumor was found under the periosteum. The tumor was well defined and was completely removed from the surrounding parietal bone. The dura under the tumor appeared normal. Cranioplasty with methylmethacrylate was performed.

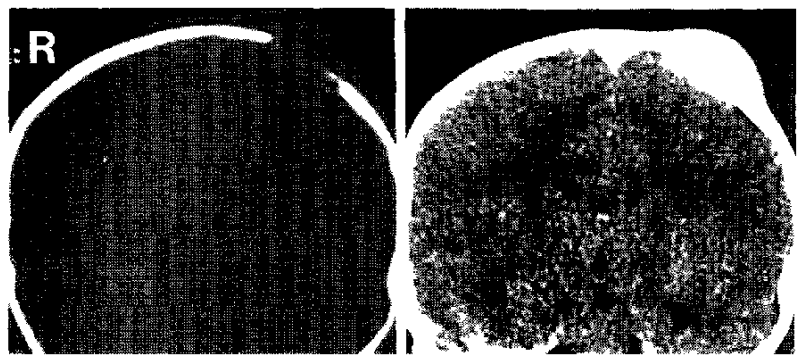

Fig. 2 left: Precontrast CT scan, coronal view, showing destruction of both the inner and outer tables of the left parietal bone. right: Postcontrast CT scan showing homogeneous enhancement of the tumor.

Macroscopically, the tumor was soft and reddish gray, with a liquefied hematoma with clots in the tumor capsule. Histological examination showed numerous red blood cells and deposits of hemosiderin (Fig. 4 upper). The tumor cells were composed of eosinophils and histiocytes in addition to many Langhans' giant cells. The histiocytes and Langhans' giant cells were positive to immunohistochemical staining for S-100 protein. Collagenous and granulomatous tissue with a number of small vessels was seen in the peripheral area of the tumor (Fig. 4 lower). The histological diagnosis was eosinophilic granuloma.

His postoperative course was uneventful and he

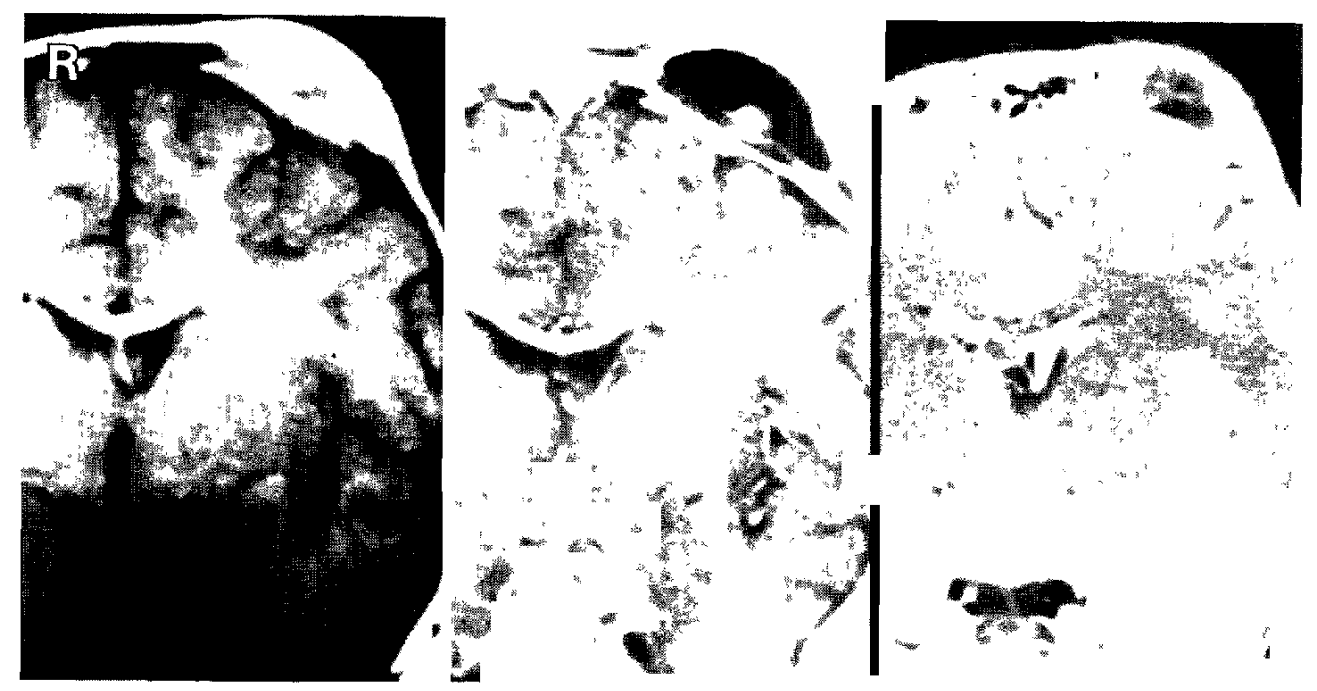

Fig. 3 left: $T_{1}$-weighted MR image showing the slightly heterogeneous high intensity mass in the left parietal region. center: $\mathrm{T}_{2}$-weighted $\mathrm{MR}$ image disclosing the hyperintense mass with partial low intensity core. right: $\mathrm{T}_{1}$-weighted MR image with Gd-DTPA showing the ring-like enhancement and compression of the underlying cortex by the tumor. 


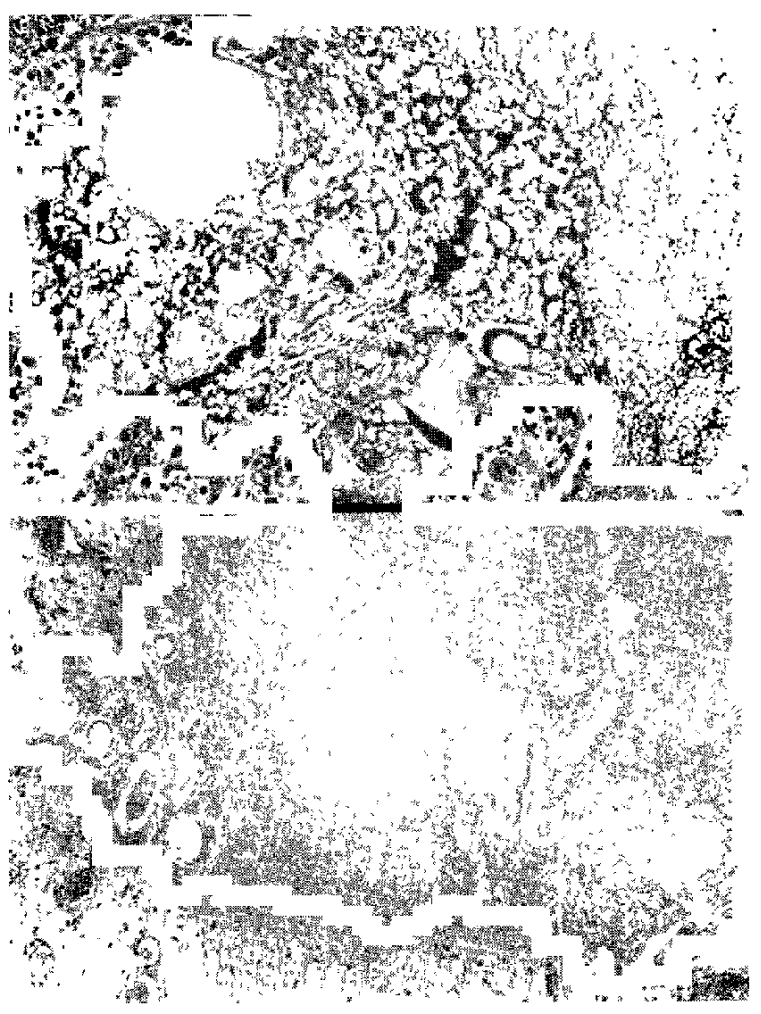

Fig. 4 Photomicrographs of the surgical specimen showing numerous Langhans' giant cells and eosinophils surrounded by red blood cells (upper: HE stain, $\times 400$ ), and a number of small vessels with fibrous collagenous tissue in the peripheral area of the tumor (lower: HE stain, $\times 100$ ).

was discharged without neurological deficit.

\section{Discussion}

Eosinophilic granuloma normally has a benign and slowly progressive course. The rapid expansion of the lesion seen in the present patient was due to the intratumoral bleeding. The incidence of intratumoral hemorrhage in central nervous system tumors is 1$11 \% .^{2,10,11)}$ The tumors most susceptible to hemorrhage are malignant types such as metastatic tumor and glioblastoma multiforme, although pituitary adenoma has the highest incidence of intratumoral bleeding. ${ }^{10)}$ The proposed mechanisms of intratumoral hemorrhage are: vascular occlusion or necrosis due to proliferation of endothelial cells of the tumor, rupture of fragile tumoral vessels, disappearance or reduction of the supportive tissue surrounding the tumoral vessels, and infiltration of tumor cells into vessel walls. ${ }^{11)}$ No tumor stain or abnormal vessels were detected in our patient, but histological examination found granulation tissue adjacent to the hemorrhage with numerous small vessels, which may have caused the bleeding tendency. In addition, there was little supportive tissue in the interstitial space within the tumor, so the hematoma could have developed readily once bleeding began.

The radiological findings of eosinophilic granuloma are well known and the diagnosis can be based on the characteristic features in most cases. Skull radiography shows a round to oval osteolytic lesion with a relatively clear non-sclerotic margin, the socalled punched-out feature. ${ }^{3)} \mathrm{CT}$ is useful for providing better delineation of the exact extent of the lesion in the adjacent soft tissues in addition to the destruction of the affected bone. ${ }^{3)}$ Postcontrast CT demonstrates homogeneous enhancement, although ringlike enhancement is also reported. ${ }^{7}$ Peripheral outer enhancement and central increased density after contrast material administration may represent opacification of granulation tissue. ${ }^{7)}$ The features of MR imaging are less well known. ${ }^{1)}$ David et al. ${ }^{3)}$ reported slight hypointensity on $T_{1}$-weighted images and mild heterogeneous hyperintensity on $\mathrm{T}_{2}$-weighted images. In our case, the tumor was heterogeneously hyperintense on the $T_{1}$-weighted images and had a hypointense core within a high intensity lesion on the $\mathrm{T}_{2}$-weighted images. Administration of Gd-DTPA enhanced the lesion to a ring-like shape. The MR imaging signal intensity suggests that the intratumoral hemorrhage in our case was in the subacute stage, with the hyperintense region on the $\mathrm{T}_{1}$-weighted image consisting of free methemoglobin. The hypointense core on $\mathrm{T}_{2}$-weighted image was consistent with intact methemoglobin within the clot. This was consistent with the findings at surgery.

The diagnosis of eosinophilic granuloma is not difficult because of the characteristic radiological and clinical features including patient's age, affected site, and clinical course. However, a rapidly enlarging mass lesion of the scalp is suggestive of malignant tumors such as neuroblastoma, osteosarcoma, and/ or chondrosarcoma. We could not make a final diagnosis until the surgical specimen was obtained. Eosinophilic granuloma should be considered in the differential diagnosis when a scalp mass lesion exhibits rapid growth.

\section{References}

1) Atlas SW: Magnetic Resonance Imaging of the Brain and Spine. New York, Raven Press, 1991, pp 353355

2) Bitoh S, Hasegawa H, Ohtsuki H: Cerebral ne- 
oplasms initially presenting with massive intracerebral hemorrhage. Surg Neurol 22: 57-62, 1984

3) David R, Oria RA, Kumar R, Singleton EB, Lindell MM, Shirkhoda A, Madewell JE: Radiologic features of eosinophilic granuloma of bone. $A J R A m J$ Roentgenol 153: 1021-1026, 1989

4) Kuwabara S, Takahashi M: Eosinophilic granuloma of the skull in identical twins. Case report. Neurol Med Chir (Tokyo) 30: 1043-1046, 1990

5) Lichtenstein L: Histiocytosis X. Integration of eosinophilic granuloma of bone, "Letterer-Siwe disease," and "Schuller-Christian disease" as related manifestations of a single nosologic entity. Arch Path (Chicago) 56: 84-102, 1953

6) Martinez-Lage JF, Poza M, Cartagena J, Vicente JP, Biec F, Heras M: Solitary eosinophilic granuloma of the pediatric skull and spine. Childs Nerv Syst 7: 448451,1991

7) Mitnick JS, Pinto RS: Computed tomography in the diagnosis of eosinophilic granuloma. $J$ Comput Assist Tomogr 4: 791-793, 1980
8) Rawlings CE, Wilkins RH: Solitary eosinophilic granuloma of the skull. Neurosurgery 15: 155-161, 1984

9) Slater JM, Swarm OJ: Eosinophilic granuloma of bone. Med Pediatr Oncol 8: 151-164, 1980

10) Wakai S, Yamamoto K, Manaka S, Takakura K: Spontaneous intracranial hemorrhage caused by brain tumor: Its incidence and clinical significance. Neurosurgery 10: 437-444, 1982

11) Yoshioka $H$, Inagawa $T$, Katoh $Y$, Tokuda $Y$, Ohbayashi N, Shibukawa M: A case of meningioma associated with intracerebral hemorrhage and acute deterioration. No Shinkei Geka 23: 79-84, 1995 (in Japanese)

Address reprint requests to: R. Uranishi, M.D., Department of Neurosurgery, Nara Medical University, 840 Shijo-cho, Kashihara, Nara 634, Japan. 